\title{
Observations of radio stars at the Valinhos CCD Meridian Circle
}

\author{
P.A.A. Lopes ${ }^{1}$, A.H. Andrei ${ }^{1}$, S.P. Puliaev ${ }^{1,2}$, J.L. Penna ${ }^{1}$, R. Teixeira ${ }^{3}$, P. Benevides-Soares ${ }^{3}$, \\ M. Assafin ${ }^{4}$, J.F. Le Campion ${ }^{5}$, and Y. Réquième ${ }^{5}$ \\ 1 Observatório Nacional/CNPq, Brasil \\ 2 Pulkovo Observatory, Russia \\ 3 Instituto Astronômico e Geofísico/USP, Brasil \\ 4 Observatório do Valongo/UFRJ, Brasil \\ 5 Observatoire de Bordeaux, France
}

Received October 27, 1998; accepted February 18, 1999

\begin{abstract}
We present the astrometric results from the optical observation of 16 stars from the list of Wendker (1995), with detected or suspected radio emission. The results are given on the Hipparcos/Tycho reference frame. The stars are evenly distributed in right ascension and the declinations range from $-15.6^{\circ}$ to $+28.6^{\circ}$. This distribution, also allowed to obtain positions relative to the Twin Astrographic Catalogue (TAC 1.0).

The observations were carried out with the Valinhos CCD Meridian Circle, in Brazil, operating in drift scanning mode. The average positional precision is at 40 mas and generally below 2 mas/yr for proper motions, obtained with the new reductions of the Astrographic Catalogue AC2000, as first epoch.

In the strips taken from the observation of the 16 radio stars, 573 Tycho stars, 545 ACT stars and 566 TAC stars were found. The analysis of the independent reductions made relatively to those frames and the large number of common stars enables to focus on the equatorial zone, as represented by the three catalogues. As a result, the quality of Valinhos observations with regard to ACT and TAC internal accuracies is verified ${ }^{1}$.
\end{abstract}

Key words: astrometry — reference systems — radio stars

Send offprint requests to: P.A.A. Lopes, Observatório Nacional/CNPq, R. Gal. José Cristino 77, CEP20921-400, Rio de Janeiro, Brasil. e-mail: afranio@on.br

1 Table 1 of this paper, plus 16 additional tables with observed positions for the ACT reference stars, are available in electronic form at the CDS via anonymous ftp to cdsarc.u-strasbg.fr (130.79.128.5) or via http://cdsweb.ustrasbg.fr/Abstract.html

\section{Introduction}

The determination of the position of radio emitting stars in the Hipparcos reference frame (ESA 1997), as represented by the Tycho (ESA 1997) catalogue and the ACT (Urban et al. 1998a) system of proper motions, enables, in principle, to study the relative orientation between that frame and the International Celestial Reference Frame ICRF (Kovalevski et al. 1997). Radio stars represent an important and unique class of objects that is directly observable by the primary instruments that define the radio and optical frames (Lindegren \& Kovalevsky 1995; Stone 1997). The advantage of using bright stars, whose radio positions are directly tied to nearby ICRF sources, however, is hampered by the errors of the stars' proper motions (Andrei et al. 1995). Therefore the interest on the high-precision observations of radio emitting stars belonging to the Hipparcos reference frame is justified. They must be made on a continuing basis leading to refinement of the proper motions.

Densification of the Hipparcos reference frame, on the other hand, is in itself desired, since many observing programs require fainter and more numerous reference stars than those in the Tycho catalogue. The Twin Astrographic Catalog - TAC (Zacharias et al. 1996) represents an important step in this direction. In the present paper, its initial version (TAC 1.0) is used. A further version shall present an improved set of proper motions and is going to be oriented in the Hipparcos system. We point however that the epochs closeness between the considered catalogues and our observations decreases the errors due to faulty proper motions. Thus we can extract from our data, a sample of the internal accuracy for the TAC in the observed zone.

At the IAG/USP CCD Meridian Circle, in Valinhos, São Paulo $\left(\varphi=-23^{\circ} 00^{\prime} 06^{\prime \prime}, \lambda=+3^{\mathrm{h}} 07^{\mathrm{m}} 52.2^{\mathrm{s}}\right.$, $h=850 \mathrm{~m}$ ), a program for the observation of 640 confirmed or suspected radio stars, taken from the list of 
Wendker (1995), is being currently run. The selected stars are contained in the declination zone $-77^{\circ}$ to $+30^{\circ}$ and in the magnitude range from 9 to 14 . The instrument has an aperture of $0.19 \mathrm{~m}$ and focal length of $2.59 \mathrm{~m}$. It is equipped with a Thomson 7895 A CCD camera, which has a $512 \times 512$ pixels matrix. The pixels are squares of $19 \mu \mathrm{m}$ side, that correspond to 1.5 arcsec on the sky. The observations are proceeded in drift scanning mode, that is, leaving the field parading across the matrix and integrating the resulting signal. The equivalent exposure time is $51^{\mathrm{s}} \sec \delta$. All the observations have been made on fields of 13 arcmin in declination by 30 minutes in right ascension, enabling an average of 36 Tycho stars and a few Hipparcos stars in each field. The detection of objects is made at the level of 3 sigma above the sky background. The center of the objects are then found by a two-dimensional Gaussian fit. The reduction of the $(x, y)$ so obtained is made through a 3 constants model for each direction independently. A comprehensive account of the instrument capabilities and mode of operation is given by Viateau et al. (1999).

We present the results from the observation of 16 stars, all within the TAC range of declinations. Of these stars, 8 belong to the TAC catalogue, 11 belong to the Tycho catalogue and 8 to the Hipparcos catalogue. Thus, 12 stars appear in at least one of the catalogues and 5 in all three. In the observed fields 573 Tycho stars and 566 TAC stars are found and also have their positions re-measured relatively to all three frames.

Finally, positions from the new reduction of the Astrographic Catalogue (Urban et al. 1998b) were searched for the radio stars, leading to a new determination of their proper motions.

\section{Data reduction}

Treatment of the data is accomplished in three stages. First, the primary data is acquired and saved to image files, usually of quite large sizes. Second, the images are located and identified, and the centering is done to produce $(x, y)$ files. Finally, the set of files covering the aimed object are reduced.

The plate overlap concept (Eichhorn \& Williams 1963) is adopted for the least squares adjustment of a three constants model to both coordinates observed values. The model is simply a polynomial of first degree in $x$ and $y$. The solution is obtained by an iterative procedure (Teixeira et al. 1992). The iteration step consists in the conventional reduction of separated tours, followed by the averaging of the residuals for the objects present in more than one tour and subtraction from the observed values.

The iteration proceeds at a fast pace and, as a rule, attains a satisfactory point after three to five steps. Except for the origin, scale and alignment, the solution is, in principle, independent from the adopted reference catalogue, as shown in Fig. 1.
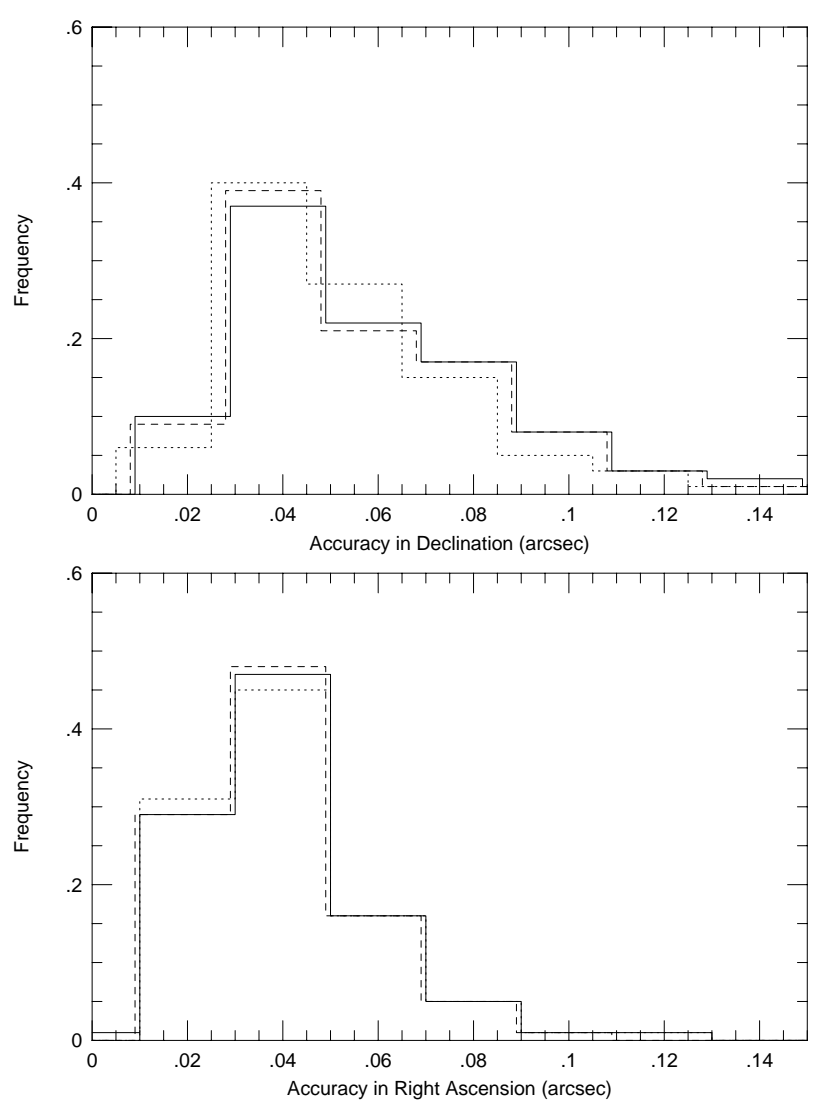

Fig. 1. Right ascension and declination frequency distribution for standard error. The reference stars with more than two used observations contribute to the plot; that is 409 ACT, 447 TAC and 397 Tycho stars. ACT solution is displayed by the dashed line, Tycho solution by the solid line and TAC solution by the dotted line

For the present sample of observations, the internal standard error for a single tour amounted to 0.'04 for both coordinates and for objects within the optimal magnitude range interval, say $V=9$ to 13 . It increases steadily for fainter magnitudes, attaining 0 "' 15 for $V=15.5$.

\section{Data analysis}

Although the internal accuracy of the measurements is not affected, to first order, by the number of reference stars, the orientation of the obtained local frame would be. This was shown by the experimental solutions based on Hipparcos stars only. To obtain a sufficient number of those, it would be required to extend the observation too long, risking to be trapped by anomalous refraction. Therefore, the reductions were made relatively to the three densest, precise catalogues, ACT, Tycho and TAC. We point that due to the inhomogeneity of TAC, one field (of the star UX Com) could not be reduced, because there were only a few (3) reference stars on it.

Figure 2 compares the observed minus catalogue offsets. All distributions display a normal characteristic. 

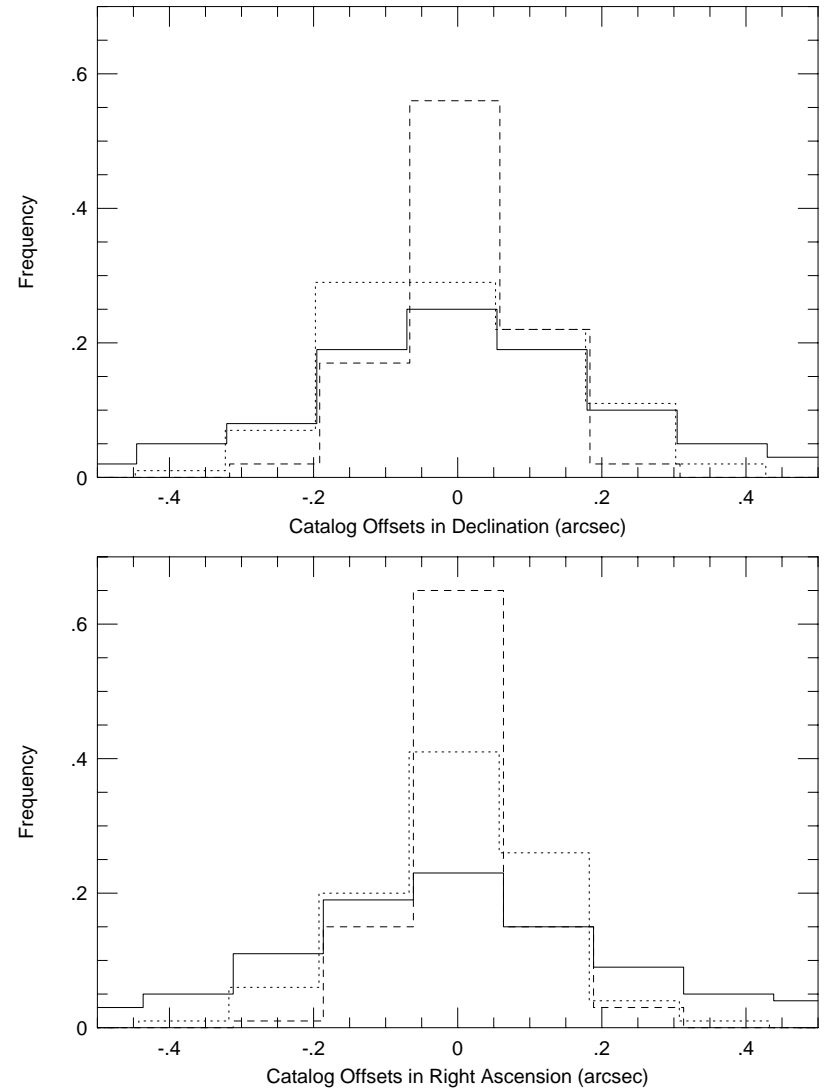

Fig. 2. Right ascension and declination observed minus catalogue distribution. The reference stars with more than two used observations contribute to the plot as in Fig. 1. ACT solution $\left(\sigma_{\alpha}=00^{\prime \prime} 09 ; \sigma_{\delta}=0\right.$. .'11) is displayed by the dashed line, Tycho solution $\left(\sigma_{\alpha}=00^{\prime \prime} 29 ; \sigma_{\delta}=0.26\right)$ by the solid line and TAC solution $\left(\sigma_{\alpha}=0\right.$ "' $13 ; \sigma_{\delta}=0$.' 16$)$ by the dotted line, with $\sigma$ being the standard-deviation

The ACT histogram (dashed line) shows the largest degree of peakness, while the Tycho histogram (solid line) shows the most pronounced side tails. The kurtosis follows the degree of proper motion precision. The TAC histogram (dotted line) confirms the inherent precision of the catalogue data and the good quality of its supplement of proper motions.

In Fig. 3 the distributions of the standard error on the final position of the program radio stars are presented, according to magnitude, number of field reference stars and number of observations. The distributions are only presented relatively to the ACT reductions, since the results for the other catalogues are similar (as shown by Fig. 1).

Within the instrumental optimal range of magnitude there is no apparent variation of precision. The noticeable exception is HBC652, in the outskirts of the optimal range. We must however, point that for HBC652 there were also a small number of reference stars and observed nights. There appears to be no loss of precision when the number of reference stars is larger than a tens or so. The largest errors in the plot pertain to HBC652, discussed
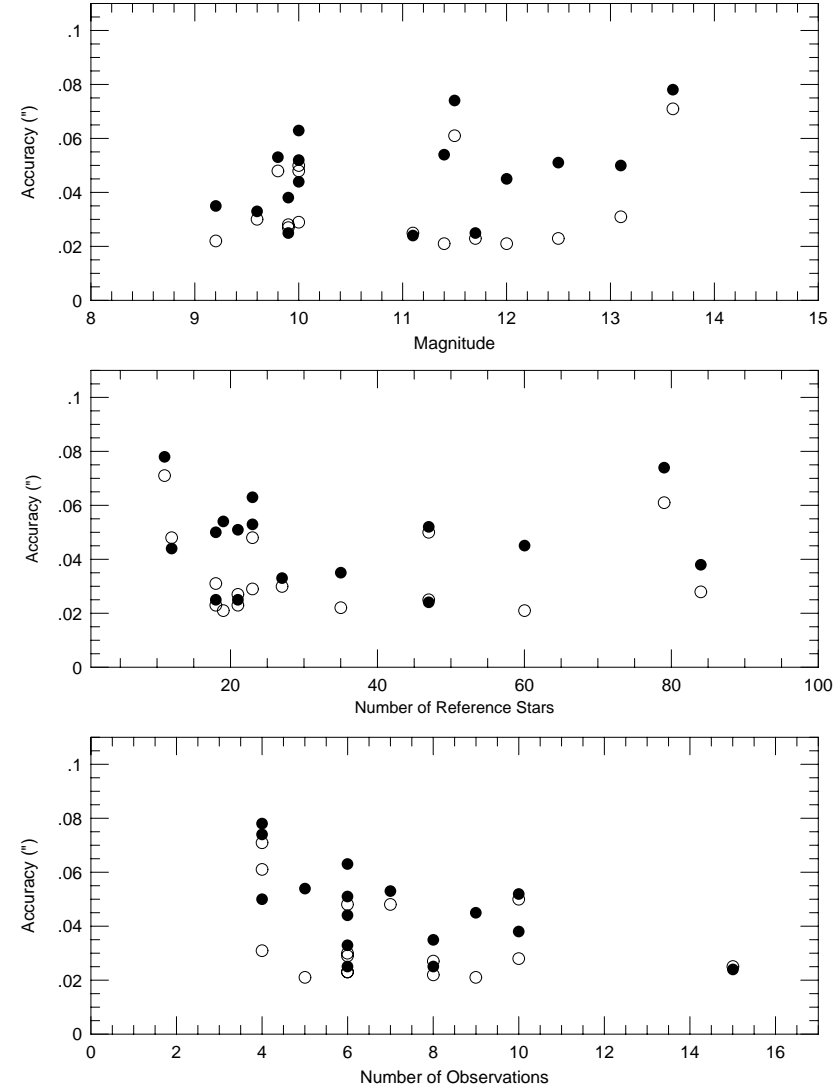

Fig. 3. Error distribution. Open circles refer to right ascension and filled circles to declination

above. Finally, it seems that usually 5 observations are sufficient to attain good internal precision, without the need of a numerous series.

As also apparent in Figs. 1 and 2, right ascensions are, in general, slightly better determined than declinations.

\section{Astrometric results}

The observed positions, referred to the ACT catalogue, are presented in Table 1. The same data referred to the Tycho and TAC catalogues are contained in Table 2. The stars are relatively well spread in right ascension, while occupying an equatorial belt.

There is a fine agreement between the Tycho and ACT referred positions, which merely derives from the fact that the ACT second epoch positions are taken from Tycho and both the Tycho and CCD observations were made at a relatively short time interval from each other. The offsets (arithmetic mean and its standard error), in the sense ACT minus Tycho, for the observed radio stars positions are: $-47 \pm 35$ mas (in right ascension) and $+15 \pm 19$ mas (in declination).

Three independent reductions are compared in Fig. 4, which displays the differences with respect to the average among the three results, for the 16 radio stars. 
Table 1. Astrometric results referred to the ACT catalogue. $N$ is the number of independent observations and $N_{\mathrm{r}}$ the number of reference stars

\begin{tabular}{|c|c|c|c|c|c|c|c|c|c|}
\hline Star & Tycho Id. & Mag & Epoch & $N$ & $\alpha(\mathrm{h}, \mathrm{m}, \mathrm{s})$ & $\varepsilon \alpha(\mathrm{s})$ & $\delta\left({ }^{\circ, \prime, \prime \prime}\right)$ & $\varepsilon \delta\left({ }^{\prime \prime}\right)$ & $N_{\mathrm{r}}$ \\
\hline HZ 1538 & & 12.0 & 1997.2 & 9 & $\begin{array}{lll}03 & 47 & 43.102\end{array}$ & 0.002 & +241655.39 & 0.05 & 60 \\
\hline WY Cnc & 1953395 & 9.6 & 1997.3 & 6 & $0901 \quad 55.455$ & 0.002 & +264122.91 & 0.03 & 27 \\
\hline AL Leo & 14151312 & 10.0 & 1997.1 & 6 & $\begin{array}{lll}09 & 58 & 12.905\end{array}$ & 0.003 & $+18 \quad 1728.07$ & 0.04 & 12 \\
\hline Gl 402 & 261224 & 11.7 & 1997.4 & 6 & $1050 \quad 52.175$ & 0.002 & +064831.46 & 0.03 & 18 \\
\hline FL Vir & & 12.5 & 1997.3 & 6 & 123317.705 & 0.002 & +09 0115.24 & 0.05 & 21 \\
\hline DT Vir & 886839 & 9.8 & 1996.5 & 7 & $1300 \quad 46.723$ & 0.003 & +122232.79 & 0.05 & 23 \\
\hline UX Com & 19951072 & 10.0 & 1997.2 & 6 & $\begin{array}{lll}13 & 0133.028\end{array}$ & 0.002 & +283754.20 & 0.06 & 23 \\
\hline BH Vir & $4968 \quad 569$ & 9.9 & 1996.5 & 8 & $13 \quad 58 \quad 24.857$ & 0.002 & -013938.90 & 0.03 & 21 \\
\hline Gl 555 & 5572804 & 11.4 & 1996.3 & 5 & 143416.898 & 0.001 & $-1231 \quad 12.53$ & 0.05 & 19 \\
\hline UZ Lib & $5595 \quad 180$ & 9.2 & 1996.6 & 8 & $15 \quad 32 \quad 23.203$ & 0.001 & $\begin{array}{lll}-08 & 32 & 00.97\end{array}$ & 0.04 & 35 \\
\hline V818Sco & & 13.1 & 1997.1 & 4 & 161955.080 & 0.002 & -153825.06 & 0.05 & 18 \\
\hline HBC652 & & 13.6 & 1996.5 & 4 & $\begin{array}{lll}16 & 48 & 18.007\end{array}$ & 0.005 & -141115.68 & 0.08 & 11 \\
\hline HM Sge & 16021636 & 11.5 & 1996.5 & 4 & 194157.075 & 0.004 & +164439.83 & 0.07 & 79 \\
\hline W Del & $1637 \quad 581$ & 9.9 & 1997.1 & 10 & $\begin{array}{lll}20 & 37 & 40.086\end{array}$ & 0.002 & +181703.76 & 0.04 & 84 \\
\hline W Equ & & 11.1 & 1996.5 & 15 & $21 \quad 15 \quad 52.624$ & 0.002 & +122319.24 & 0.02 & 47 \\
\hline EQ Peg & 1723 & 10.0 & 1996.7 & 10 & $23 \quad 3152.071$ & 0.004 & +195614.32 & 0.05 & 47 \\
\hline
\end{tabular}

Table 2. Astrometric results referred to the Tycho and TAC catalogues. The number of observations, mean epoch, hours and minutes of right ascension and degrees and minutes of declination follow those on Table $1 . N_{\mathrm{r}}$ is the number of reference stars. The TAC position for UX Com could not be determined

\begin{tabular}{lcccccccccc}
\hline Star & $\alpha$ Tyc & $\varepsilon \alpha(\mathrm{s})$ & $\delta_{\text {Tyc }}\left({ }^{\prime \prime}\right)$ & $\varepsilon \delta\left({ }^{\prime \prime}\right)$ & $N_{\mathrm{r}}$ & $\alpha$ TAC $(\mathrm{s})$ & $\varepsilon \alpha(\mathrm{s})$ & $\delta_{\text {TAC }}\left({ }^{\prime \prime}\right)$ & $\varepsilon \delta\left({ }^{\prime \prime}\right)$ & $N_{\mathrm{r}}$ \\
\hline HZ 1538 & 43.113 & 0.002 & 55.40 & 0.04 & 63 & 43.102 & 0.002 & 55.47 & 0.05 & 42 \\
WY Cnc & 55.455 & 0.002 & 22.97 & 0.04 & 28 & 55.450 & 0.002 & 22.51 & 0.03 & 13 \\
AL Leo & 12.915 & 0.003 & 28.06 & 0.05 & 13 & 12.888 & 0.004 & 28.12 & 0.05 & 12 \\
Gl 402 & 52.166 & 0.001 & 31.35 & 0.03 & 19 & 52.170 & 0.002 & 31.12 & 0.03 & 20 \\
FL Vir & 17.701 & 0.001 & 15.18 & 0.05 & 21 & 17.697 & 0.002 & 15.00 & 0.05 & 35 \\
DT Vir & 46.729 & 0.003 & 32.84 & 0.05 & 24 & 46.713 & 0.003 & 32.70 & 0.05 & 33 \\
UX Com & 33.039 & 0.002 & 54.11 & 0.06 & 23 & - & - & - & - & - \\
BH Vir & 24.856 & 0.002 & 38.92 & 0.02 & 21 & 24.851 & 0.002 & 39.13 & 0.03 & 39 \\
Gl 555 & 16.892 & 0.001 & 12.54 & 0.06 & 20 & 16.905 & 0.001 & 12.58 & 0.06 & 35 \\
UZ Lib & 23.198 & 0.001 & 00.88 & 0.04 & 36 & 23.205 & 0.002 & 00.97 & 0.04 & 53 \\
V818Sco & 55.102 & 0.002 & 25.27 & 0.05 & 19 & 55.075 & 0.002 & 25.26 & 0.05 & 36 \\
HBC652 & 18.031 & 0.005 & 15.76 & 0.08 & 12 & 18.022 & 0.005 & 15.67 & 0.08 & 14 \\
HM Sge & 57.077 & 0.004 & 39.81 & 0.07 & 82 & 57.066 & 0.004 & 39.74 & 0.08 & 81 \\
W Del & 40.087 & 0.002 & 03.77 & 0.04 & 90 & 40.071 & 0.002 & 03.77 & 0.04 & 65 \\
W Equ & 52.625 & 0.002 & 19.28 & 0.02 & 52 & 52.617 & 0.002 & 19.12 & 0.02 & 45 \\
EQ Peg & 52.070 & 0.003 & 14.34 & 0.05 & 50 & 52.053 & 0.003 & 14.25 & 0.05 & 43 \\
\hline
\end{tabular}

The TAC referred to positions were transported to the Hipparcos reference frame by means of the procedures given by Feissel \& Mignard (1998). It is verified that the ACT and Tycho referred results cluster together, while the results referred to the TAC stand noticeably apart from the other two. This is in line with the Hipparcos and FK5 reference frames differences (Argyle et al. 1996) in the concerned declination zone. For the small sample displayed in the figure, the offsets (as before), in the sense
ACT minus TAC, are: $+98 \pm 32$ mas (in right ascension) and $+113 \pm 35$ mas (in declination).

To confirm such possible systematic trend and its magnitude, we have extended the comparison to all the 415 common reference stars in the observed fields. In this case, the offsets for ACT minus TAC referred positions are: $\Delta \alpha$ $\cos \delta=+123 \pm 6$ mas $(\sigma=122$ mas $)$ and $\Delta \delta=+108$ \pm 7 mas $(\sigma=146$ mas), with $\sigma$ being the corresponding standard deviations. For comparison, the corresponding differences for the 510 common ACT and Tycho observed 
Table 3. Proper Motions derived from the ACT referred positions. The derived proper motions (Cols. 2 to 5 ) are based on the radio star positions obtained in this work and those from the AC2000 catalogue. For comparison, proper motions from the ACT are also given (Cols. 6 to 9, with the upper asterisk), as well as additional resources of optical positional data for the stars (Col. 10). All values are in mas/yr. First epoch positions for Gl 402 and HM Sge were extracted from Hipparcos and CMC4 (on Hipparcos system), respectively, for these stars are absent on the AC2000 and because those positions give minimum proper motion errors

\begin{tabular}{llrllllllc}
\hline Radio Star & $\mu_{\alpha} \cos \delta$ & Error & \multicolumn{1}{c}{$\mu_{\delta}$} & Error & $\mu_{\alpha}^{*} \cos \delta$ & Error & $\mu_{\delta}^{*}$ & Error & Catalogues \\
\hline HZ 1538 & -1.2 & 1.7 & -0.3 & 0.8 & & & & & AC \\
WY Cnc & -9.7 & 2.3 & -50.0 & 1.9 & -9.5 & 2.4 & -50.9 & 1.7 & AC,Hipp,Tyc, TAC,BORD \\
AL Leo & -6.8 & 2.1 & +0.1 & 1.8 & -6.6 & 2.0 & +0.5 & 1.5 & AC,Tyc,TAC,ACRS,PPM,AGK3U \\
Gl 402 & -876.9 & 6.3 & -806.5 & 2.3 & & & & & Hipp,CMC5 \\
DT Vir & -639.7 & 27.8 & -21.6 & 2.2 & -639.2 & 29.8 & -23.1 & 1.8 & AC,Tyc, TAC,BORD,CMC5 \\
UX Com & -59.3 & 3.0 & -2.2 & 2.8 & -59.6 & 3.3 & -2.6 & 2.4 & AC,Hipp,Tyc,BORD \\
BH Vir & +4.8 & 1.0 & -3.3 & 1.8 & +5.2 & 0.8 & -3.4 & 1.7 & AC,Hipp,Tyc,TAC,ACRS,PPM,AGK3U \\
Gl 555 & -350.8 & 1.2 & +592.8 & 3.8 & -350.0 & - & +591.8 & - & AC,Hipp,TAC,CMC4,CMC7 \\
UZ Lib & +27.2 & 1.9 & +2.9 & 5.0 & +27.3 & 1.9 & +3.8 & 5.0 & AC,Hipp,Tyc,TAC,BORD,CMC4 \\
V818 Sco & -7.7 & 2.0 & -13.0 & 2.0 & & & & & AC,BORD,CMC5 \\
HM Sge & -18.9 & 18.3 & +17.1 & 20.1 & & & & & BORD,CMC4,CMC5,Tyc \\
W Del & -5.3 & 1.6 & -2.3 & 2.0 & -5.3 & 1.4 & +2.5 & 1.8 & AC,Hipp,Tyc,TAC,BORD \\
W Equ & -12.8 & 1.2 & -21.7 & 1.5 & & & & & AC,TAC \\
EQ Peg & +545.0 & 7.1 & -42.9 & 1.1 & +541.2 & 7.6 & -42.2 & 0.9 & AC,Hipp,Tyc,BORD \\
\hline
\end{tabular}

reference stars are found smaller: $\Delta \alpha \cos \delta=-34 \pm 12$ mas ( $\sigma=260$ mas $) ; \Delta \delta=-10 \pm 9$ mas $(\sigma=208$ mas $)$. Despite this, the standard deviations are larger than those found for the ACT minus TAC differences. These results, in line with what is seen on the catalogue offsets histograms, point to the high quality of the TAC 1.0 catalogue, although they are affected by zonal warps originating from the FK5 frame.

Proper motions for the program stars were derived exclusively from the positions referred to the ACT and from the AC2000 (Urban et al. 1998b), which furnish first epoch positions. First epoch positions for Gl 402 and HM Sge were extracted from Hipparcos and CMC4 (on Hipparcos system), respectively, for these stars are absent on the AC2000 and because those positions give minimum proper motion errors. For comparison, intermediate epochs data were collected from various sources: the catalogues Hipparcos, Tycho, TAC, AGK3U (Bucciarelli et al. 1992), PPM (Röser \& Bastian 1993), ACRS (Corbin \& Urban 1991), Bordeaux (Réquième \& Mazurier 1991) and Carlsberg (Morrison 1985 through 1994). The data referred to the FK5 system were reduced to the Hipparcos according to the procedures of Feissel \& Mignard (1998).

The results are given in Table 3 and Fig. 5. The graph for HM Sge is suggestive of the occurrence of multiplicity, which could explain the relatively large declination errors for this star. No first epoch position was found for FL Vir and for HBC 652 .

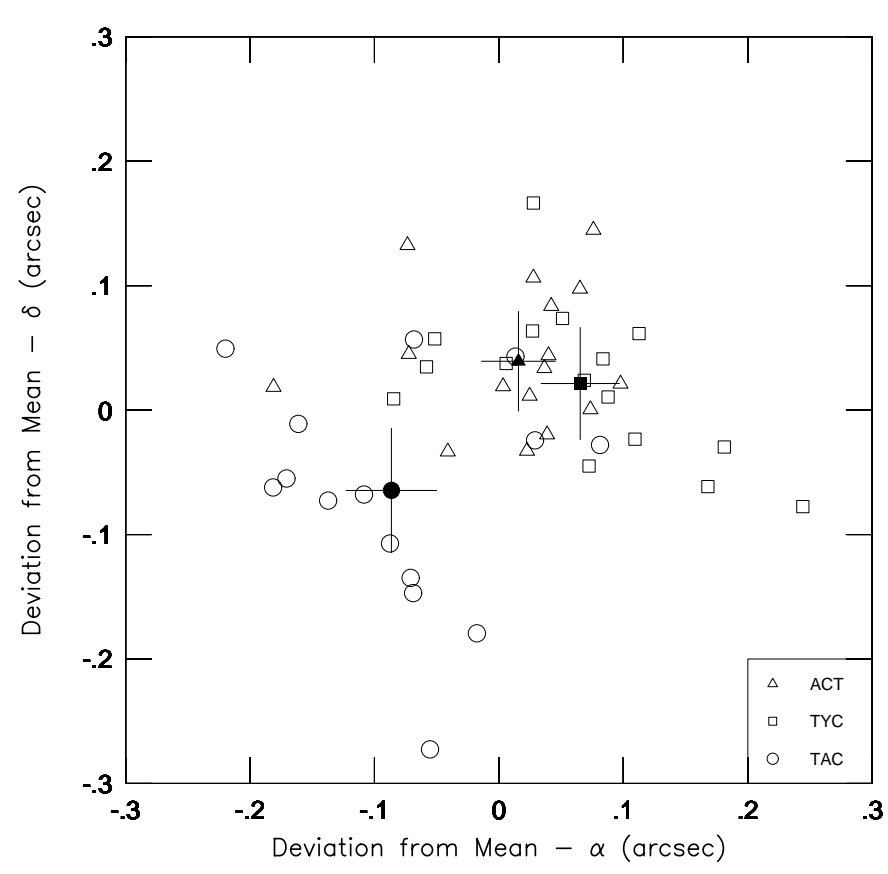

Fig. 4. Comparison of position differences: The filled symbols represent the mean positional difference between the sample of 16 radio stars for the three catalogues, respectively. Individual position differences are represented by the plain symbols. The abbreviation TYC, in the figure, is associated with the Tycho catalogue 

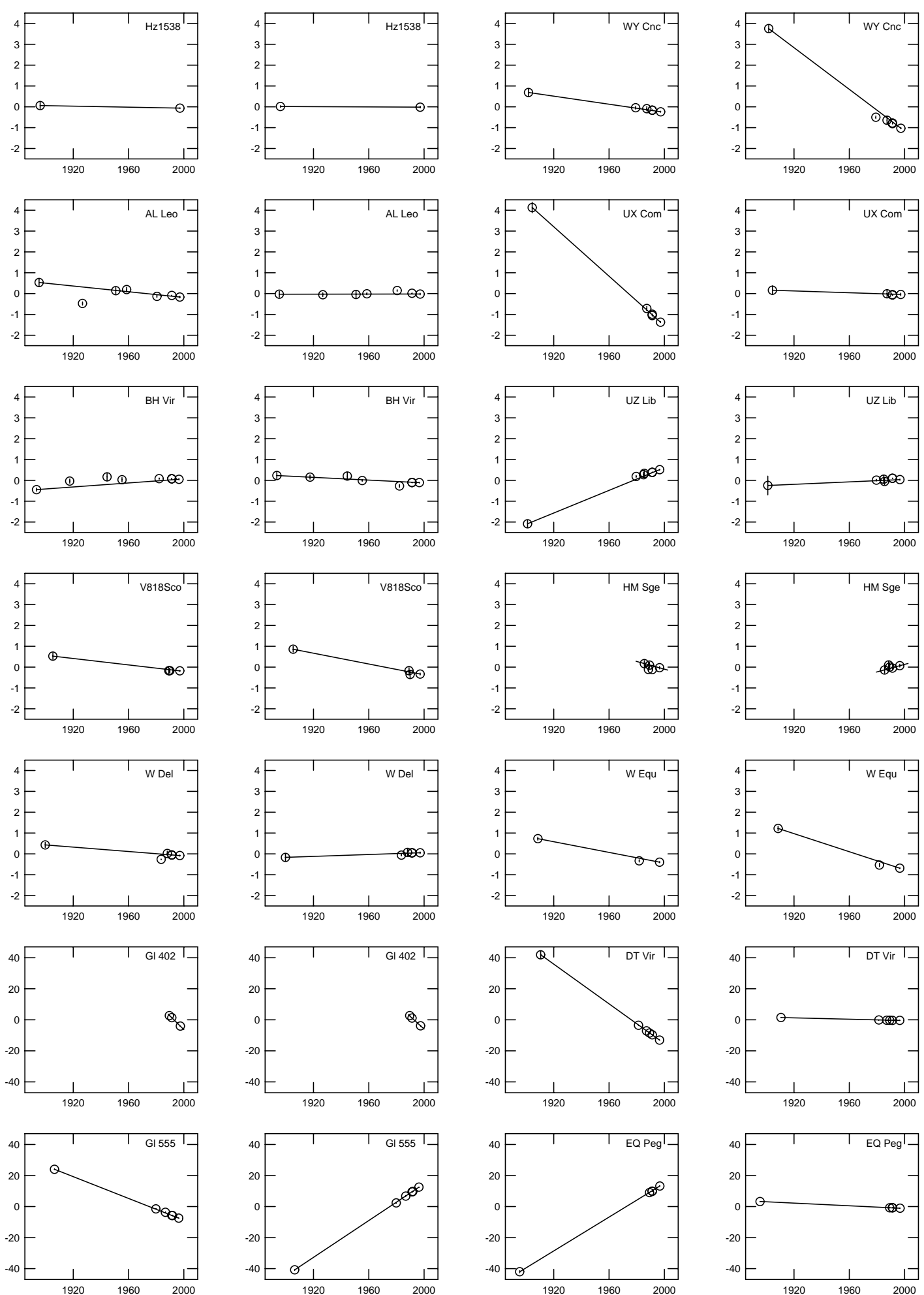

Fig. 5. Proper motions for the program radio stars, as derived from Valinhos observations and AC2000 positions. Intermediary positions from various catalogues are plotted for comparison. (See Table 3). First and third columns give right ascension proper motions, while the second and fourth columns give the declination ones. All values are in arcsec/yr. The last four plots (Gl 402, DT Vir, Gl 555 and EQ Peg) are in a different scale from the others, since they refer to high proper motion stars 


\section{Conclusions}

The positions of 16 stars with confirmed or suspected radio emission were determined on the Hipparcos/ICRS reference system, as materialized by the ACT catalogue. The corresponding positions were also determined relatively to the Tycho and TAC 1.0 catalogues (15 radio stars). The level of precision is at 35 mas for right ascensions and 46 mas for declinations. For 5 of these stars there was no previous position on the Hipparcos system.

The analysis of the results for the radio stars shows that the performance of the Valinhos CCD Meridian Circle and of the observation and reduction procedures can provide positions to an accuracy better than 50 mas to the magnitude range from 9 to 13 , provided that typically a minimum of 10 reference stars are present and 5 good nights of observation are used at least.

The proper motions for 14 of the studied radio stars are found, to an average precision better than 2 mas/year. The proper motions are found in general agreement with the ACT and TAC ones.

The scan drift mode of observation enabled to obtain accurate positions for many stars in the observed strips. In particular, positions at the observation epoch have been obtained for 545 ACT stars, 573 Tycho stars and 566 TAC stars. These results are available in electronic form.

As the TAC catalogue can play an all important role for the densification of the Hipparcos system, an investigation was carried out concerning its internal consistency. Naturally this investigation is limited by the number of observed strips (15) and the width of the declination belt (from $-15.6^{\circ}$ to $+28.6^{\circ}$; by average $\delta=+10.5^{\circ}$ ). The internal precision of the TAC referred reductions is close to the ACT ones. An average offset of 100 mas, similar for both equatorial coordinates, in the sense Hipparcos minus FK5, is found from the position differences between the common ACT and TAC reference stars. Thus, the analysis enables to confirm the TAC 1.0 catalogue qualities, moreover that only the equatorial zone was studied here, indicating that its extension on the Hipparcos system may bring an important contribution to its densification.

In all, the results obtained show that the present program shall be able to present reliable positions for a large number of radio stars, placed on the Hipparcos reference system.

Acknowledgements. We acknowledge financial support from CAPES, CNPq and FAPESP. Also we acknowledge the suggestions made by the referee, Dr. H.G. Walter.

\section{References}

Andrei A.H., Jilinski E.G., Puliaev S.P., 1995, AJ 109, 428

Argyle R.W., Einicke O.H., Pilkington J.D.H., Morrison L.V., Fabricius C., Helmer L., 1996, A\&A 312, 1031

Bucciarelli B., Daou D., Lattanzi M.G., Taff L.G., 1992, The AGK3U: an updated version of the AGK3, AJ 103, 1689

Corbin T.E., Urban S.E., 1991, Astrographic Catalogue Reference Stars, Doc. No. NSSDC/WDC-A-R \& S 91-90, Greenbelt, U.S.A.

Eichhorn H., Williams C.A., 1963, AJ 68, 221

ESA, 1997, The Hipparcos and Tycho Catalogs (ESA SP-1200)

Feissel M., Mignard F., 1998, A\&A 331, L33

Kovalevsky J., Lindengren L., Perrymam M.A.C., et al., 1997, A\&A 323, 620

Lindengren L., Kovalevsky J., 1995, A\&A 304, 189

Morrison L.V., 1985-94, Carlsberg Meridian Catalogues Numbers 1-8, Copenhagen University Observatory, Royal Greenwich Observatory and Real Instituto y Observatorio de la Armada en San Fernando

Réquième Y., Mazurier J.M., 1991, A\&ASS 89, 311

Röser S., Bastian U., 1993, PPM Star Catalogue, Bull. Inf. CDS 42, 11

Stone R., 1997, AJ 114, 1679

Teixeira R., Réquième Y., Benevides-Soares P., Rapaport M., 1992, A\&A 264, 307

Urban S.E., Corbin T.E., Wycoff G.L., 1998a, AJ 115, 2161

Urban S.E., Corbin T.E., Wycoff G.L., Martin J.C., Jackson E.S., Zacharias M.I., Hall D.M., 1998b, AJ 115,1212

Viateau B., Réquième Y., Le Campion J.F., et al., 1999, A\&AS 134,173

Wendker H.J., 1995, A\&ASS 109, 177

Zacharias N., Zacharias M.I., Douglass G.G., Wycoff G.L., 1996, The Twin Astrographic Catalog (TAC) Version 1.0, AJ 112,2336 